\title{
EXPERIENCIA DE APRENDIZAJE DE ALUMNOS DE ENFERMERÍA EN PRÁCTICA DE SALUD MENTAL Y PSIQUIATRÍA
}

\section{STUDENT LEARNING EXPERIENCE IN NURSING PRACTICE OF MENTAL HEALTH AND PSYCHIATRY}

\author{
Hernán Henríquez T.* \\ EDITH Rivas R. ** \\ VERÓNica GarCía S. ${ }^{* * *}$
}

\begin{abstract}
RESUMEN
El proceso de aprendizaje en prácticas clínicas (PC), en la formación profesional de enfermeras(os), permite adquirir habilidades, razonamientos y destrezas. Objetivo: Conocer la experiencia de aprendizaje de estudiantes de Enfermería respecto de su práctica clínica en Salud Mental y Psiquiatría. Material y método: Investigación cualitativa, por grupos focales, selección de 18 sujetos de estudio, constituyéndose 3 grupos focales de 6 integrantes cada uno. Análisis por comparación constante. En validez y confiabilidad se realizó triangulación por investigador y criterios de rigor de Guba y Lincoln. Consideraciones éticas: se cumplió con criterios de Declaración de Helsinki y firma del Formulario de Consentimiento Informado. Resultados: Emergen metacategorías: aprendizaje integral (práctica productiva, importante), rol del enfermero psiquiátrico (administrativo), influencia del modelo conductista y actitudes - creencias (miedo al inicio que cedía al finalizar la práctica). Conclusión: Se develan sensaciones negativas, miedo al inicio de la práctica clínica y se realiza aprendizaje integral.
\end{abstract}

Palabras clave: Salud mental, estudiantes de Enfermería, prácticas clínicas.

\begin{abstract}
The learning process in clinical practice $(\mathrm{CP})$ helps trainee nurses to acquire practical and theoretical skills. Objective: To know the learning experience of Nursing students regarding their $\mathrm{CP}$ in mental health and psychiatry. Methods: Qualitative research using focus groups, selection of 18 study subjects arranged in 3 focus groups of 6 members. Use of constant comparative analysis. In relation to validity and reliability the analysis was carried out using investigator triangulation and Lincoln's and Guba's evaluative criteria. Ethical principles of the Declaration of Helsinki and signing of an Informed Consent Form were considered. Results: Metacategories such as integrated learning (productive practice), role of the psychiatric nurse (administrative), influence of behavioral model and attitudes-beliefs (initial clinical anxiety). Conclusion: Negative feelings, initial clinical anxiety and comprehensive learning.
\end{abstract}

Key words: Mental health, students, nursing, clinical clerkship.

Fecha recepción: 03/06/15 Fecha aceptación: 27/12/16

\footnotetext{
*Enfermero. Docente Departamento de Enfermería Universidad de La Frontera. Temuco, Chile. E-mail: hhenriquezt@hotmail.com

${ }^{* *}$ Enfermera. Docente Departamento de Enfermería. Universidad de La Frontera. Temuco, Chile. E-mail: edith.rivas@ ufrontera.cl

${ }^{* * *}$ Enfermera. Hospital Dr. Hernán Henríquez Aravena. Temuco, Chile. E-mail: verogarcia777@gmail.com
} 


\section{INTRODUCCIÓN}

Epidemiológicamente, los indicadores de salud mental y psiquiatría (1) expresan que una de cada cuatro personas en el mundo sufrirá una patología psiquiátrica en el transcurso de su vida, iniciándose antes de los 14 años. La Asociación Americana de Enfermeras Psiquiátricas indica que hay una escasez de enfermeras en todos los niveles y que motivar a una generación de enfermeras a que deseen trabajar con personas con trastornos de salud mental es un reto para los educadores de Enfermería (2).

En Chile se estima que un tercio (36\%) de la población chilena sufrirá una enfermedad de salud mental en el transcurso de su vida y un poco más de la quinta parte de la población $(22,5 \%)$ ha presentado un trastorno de salud mental en los últimos doce meses (3). En este contexto, el Ministerio de Salud de Chile observa con preocupación el problema, a pesar de contar con un programa destinado a atender a los usuarios que presenten estas patologías (4).

La magnitud de estos antecedentes pone de manifiesto la significación y trascendencia de esta área clínica en la formación de las/los enfermeras/os, ya que una experiencia positiva supondrá que los estudiantes vean la enfermería de salud mental y el cuidado de las personas con enfermedad mental de manera más positiva $(5,6)$.

La revisión de la literatura da cuenta de investigaciones que describen la práctica clínica, en Brasil, como una experiencia positiva (7), de igual forma en Australia (8), estudiantes refieren haberse sentido bienvenidos, bien orientados y apoyados durante su experiencia clínica en salud mental (5). Artículos publicados en México develan en los estudiantes sentimientos de temor, preocupación, miedo, ansiedad y nerviosismo (9). Una investigación chilena concluye que los estudiantes conciben las prácticas clínicas como una metodología activa importante para su formación profesional (10). Finalmente un estudio sobre la atención de salud mental por el equipo de enfermería revela que, a pesar de los avances realizados por la reforma psiquiátrica, se sigue observando rasgos de enfoque reduccionista en la especialidad, reflejando una práctica clínica que no considera al sujeto enfermo. Enfoques que se perciben en la práctica de enfermería en los hospitales y en los centros de Atención Psicosocial (11).

Los antecedentes presentados dieron lugar a la siguiente interrogante: ¿Cuál es la experiencia de aprendizaje de los estudiantes de Enfermería de una universidad chilena, respecto de sus Prácticas Clínicas en el Servicio de Psiquiatría de un Hospital de alta complejidad? De esta manera el objetivo del estudio fue conocer la experiencia de aprendizaje de estudiantes de Enfermería respecto de su práctica clínica en Salud Mental y Psiquiatría.

Así, se plantea un problema de naturaleza educativa que se circunscribe a las opiniones narradas por los alumnos respecto del proceso enseñanza-aprendizaje en las Prácticas Clínicas (PC) en un Servicio de Salud Mental y Psiquiatría. Se espera que este estudio aporte antecedentes que contribuyan a la formación de las/los estudiantes de Enfermería en el área de salud mental y psiquiatría.

\section{MATERIAL Y MÉTODO}

La investigación se apoyó en el paradigma cualitativo, con diseño fenomenológico. La unidad de análisis fue el estudiante de tercer año de la Carrera de Enfermería de una Universidad del sur de Chile, que cursaba práctica en un Servicio de Psiquiatría. La muestra fue intencionada de casos por criterio, conformada por 18 sujetos. Fueron considerados criterios de selección: estudiantes matriculados en tercer año de la Carrera de Enfermería, cursando PC en Psiquiatría y con disponibi- 
lidad horaria para asistencia. La recolección de datos se realizó a través de tres grupos focales de seis integrantes cada uno, quienes accedieron a participar en el estudio previa firma del Formulario de Consentimiento Informado. Se consideró la participación de dos investigadores (uno en registro de notas de campo y grabación y un segundo en rol de conductor). El lugar de realización fue una sala de reuniones, por disposición física, amplitud y comodidad; efectuadas al término de la evaluación de PC y previa autorización de la enfermera Supervisora del Servicio de Psiquiatría.

Los participantes fueron invitados a reflexionar en aspectos no tematizados (12); se efectuó un proceso objetivo, riguroso y sistemático, desde una perspectiva holística (13). El diseño fue flexible, permitiendo la interacción entre el investigador y los sujetos de estudio (14). Se proporcionó una interpretación a través de la interacción lógica con los demás y se situó el objeto en sus marcos de referencia, propia perspectiva y se codificaron los significados. De esta forma el fenómeno sometido a análisis se evaluó en diversos aspectos, dimensiones o componentes (15); fue descriptivo, al centrar los significados de las acciones humanas, comprendiendo la narrativa de los informantes claves (16).

Para el registro de la información, en las notas de campo, se registró la subjetividad del proceso (detalles, comentarios textuales, actitudes), constituidos en apuntes, para recordar la observación realizada y facilitar el estudio y reflexión (14). Las grabaciones fueron un registro fidedigno, evitando deformación o sesgos. Se contó con una pauta de entrevista para la orientación de los temas.

$\mathrm{El}$ análisis de datos respondió a una perspectiva descriptiva de la información textual transcrita desde las grabaciones, donde se buscaron tendencias o tipologías representativas del tema que pudieran dar respuestas a la pregunta de investigación; se conformaron en primera instancia categorías, luego se efectuaron revisiones progresivas, reduciendo la información a metacategoría, subcategoría y unidades semánticas. En validez y confiabilidad se utilizó triangulación por investigador, participando el investigador principal, y dos académicas con experiencia en investigación, cada uno de estos realizó un análisis individual de los datos recopilados, luego se realizó una reunión en conjunto para comparar los resultados obtenidos, siguiente a eso se llegó a acuerdos, para finalmente obtener las unidades semánticas. En criterios de rigor se trabajó con dependencia, credibilidad, auditabilidad y transferibilidad (17).

Aspectos éticos de la investigación: uno de los preceptos para realizar esta investigación fue analizar el Código de Ética del Colegio de Enfermeras de Chile (18) y la Declaración de Helsinki (19), que refiere la protección de los estudiantes que participan en el estudio; conjuntamente se contó con autorización del Comité de Ética de la universidad de estudio y Consentimiento Informado de cada uno de los participantes.

\section{RESULTADOS}

Los sujetos de estudio fueron 18 estudiantes de tercer año de la Carrera de Enfermería de una universidad chilena, 14 mujeres y 4 hombres; en rango de edad 23 y 27 años y 20 a 22 años, respectivamente.

\section{Análisis descriptivo}

En la expresión sobre experiencia de aprendizaje de las PC en salud mental y psiquiatría se develaron metacategorías y subcategorías (Figura 1).

En la metacategoría aprendizaje integral, los estudiantes consideran que es una práctica productiva, importante, humana, de alto aprendizaje y necesaria, porque se instruye a trabajar con un paciente con patología psiquiátrica, en un entorno de servicio accesible, con buena disposición y trato digno a los 
Figura 1. Experiencia de aprendizaje respecto de las prácticas clínicas en salud mental y psiquiatría.

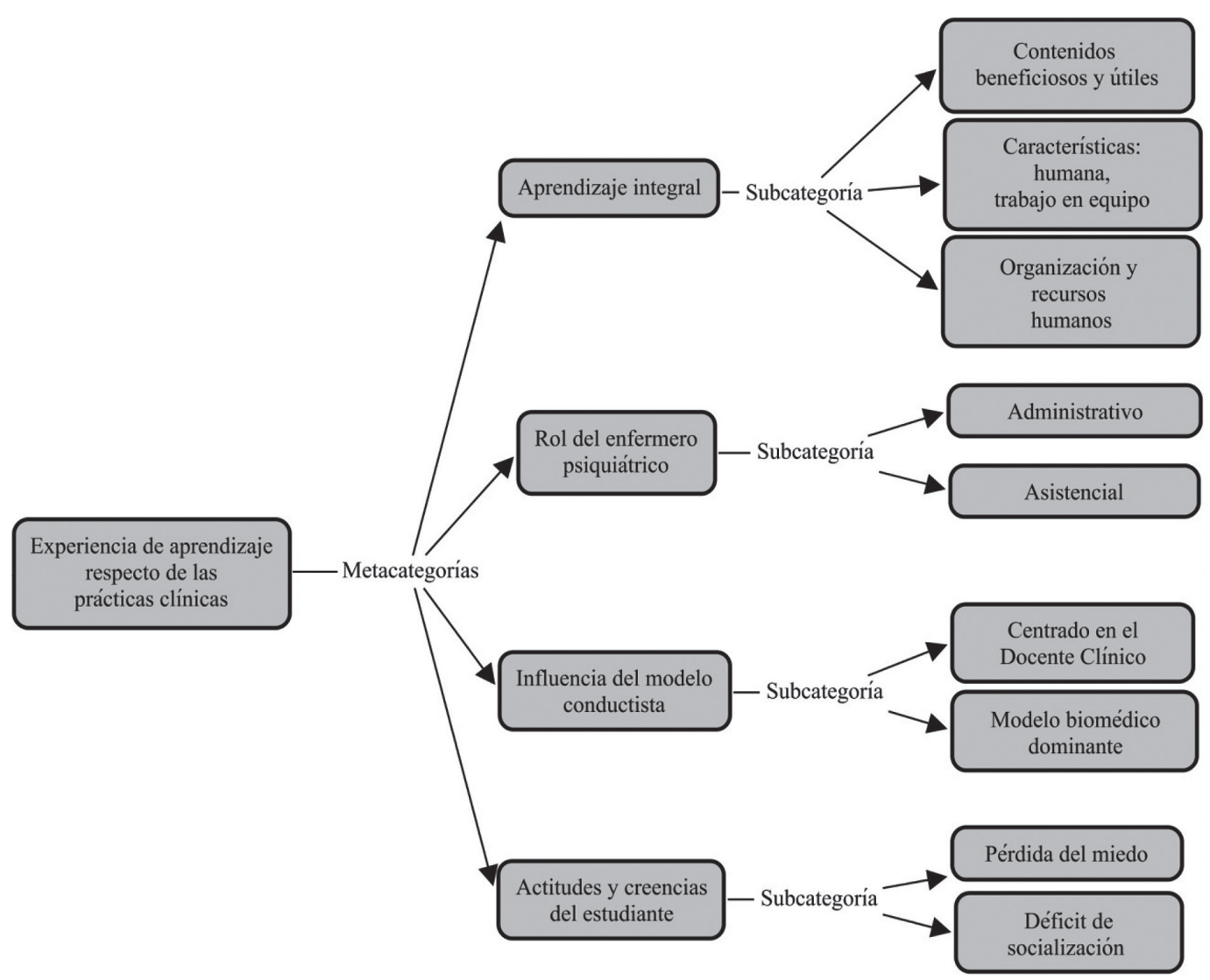

pacientes, existiendo trabajo en equipo, especialmente con los técnicos de nivel superior. Un estudiante expresa: "este es un servicio distinto a todos los demás, tiene una particularidad". En las subcategorías: Organización y recursos humanos, manifiestan que les agrada la planificación y su cronograma, por el orden de los tiempos, lo que facilita el cumplimiento de los objetivos, sin dejar de mencionar que algunas actividades no se realizaron en total orden, debido a que dependían de la presencia del docente o las indicaciones que dejara durante su ausencia. Respecto de los recursos humanos, manifiestan que el trabajo del Terapeuta Ocupacional fue entretenido: "yo no sabía de qué se trataba el rol, fue entretenido, observé el cambio de las personas, posparticipación, también el trabajo de la psicóloga, deberíamos haber visto más esa parte" y manifiestan mayor inclusión en la asignatura, con escucha activa, interacción y cómo enfrentar a este tipo de paciente. En Características manifiestan: "está en construcción, bien enfocada, pero le faltan detalles", algunos comentan que está aislada del resto, "que los detalles son abordables".

En la metacategoría: Rol del enfermero psiquiátrico, subcategoría: Administrativo, se aprecia en los relatos agrado por realizar actividades administrativas, como categorización de camas, revisión de indicaciones médicas, tarjetero de fármacos, "me gustó la parte administrativa, la categorización no la había hecho nunca, en el ámbito administrativo se destacó el rol". En la segunda subcategoría: Asistencial se puede apreciar una marcada disposición a realizar procedimientos, "no se administran medicamentos endovenosos a 
cada momento, colocar vías, la sociabilización es el enfoque, lo asistencial es conocer, saber cómo llegar, como escuchar, cómo abordar".

En la metacategoría: Influencia del modelo conductista, subcategoría: Centrado en el docente clínico, refieren que el rol es entregar el conocimiento, no se observa la visión constructivista: "me hubiese gustado que el profesor nos comentará de la esquizofrenia". Además manifiestan que en presencia del docente, el aprendizaje es más efectivo, en su ausencia no organizaban su tiempo, por tanto solicitan su disposición diaria: "yo creo que el docente hace la práctica”. Conjuntamente, se evidenció déficit en la capacidad de autoaprendizaje: "en ningún momento estudiamos Guías Clínicas: Depresión Mayor, entonces no lo aplicamos, no lo vimos, necesitamos que el docente nos pregunte e interrogue". De esta forma se aprecia que no existe conciencia respecto de la metodología (estudiante protagonista y docente un facilitador). En la subcategoría: Modelo biomédico dominante, consideran de mayor importancia las patologías clínicas y farmacología que el cuidado del paciente o el rol administrativo como se expone aquí: "a mí me interesa más la patología que el tema administrativo". Se centran en la fisiología y fármacos, solicitan ítems de patologías: "me gustaría saber qué está pasando con la dopamina".

En metacategoría: Actitudes y creencias del estudiante, subcategorías: Pérdida de miedo y déficit de sociabilización con el paciente; en la primera opinan que se produce inquietud e inseguridad al relacionarse con los pacientes; sentimientos que les provocan inquietud, posteriormente este aspecto se reduce. Respecto de la socialización, se percibe dificultad para abordar al paciente, lo que es catalogado como obstaculizador: "se nos obstaculizó un poco, porque no tenemos la habilidad y es difícil llegar a estos pacientes con estas patologías, abordar al paciente conversar con él, lo encontré complicado".

En la perspectiva global de los temas surgidos en esta investigación, se puede eviden- ciar una tendencia a expresar o considerar la PC en salud mental y psiquiatría, por parte de los estudiantes, como una práctica positiva en relación a su aprendizaje.

\section{DISCUSIÓN Y CONCLUSIÓN}

En primer término se debe establecer que la investigación plantea una perspectiva del pensar de los estudiantes, siendo ellos el eje fundamental de todo proceso educacional. Es una temática poco estudiada y específicamente en un área permeada por la vulnerabilidad social, como es la salud mental y psiquiatría.

Los resultados obtenidos caracterizan el aprendizaje como esencial en el proceso de formación profesional, similar a otros estudios en que los estudiantes consideran la experiencia clínica como única y la supervisión como favorable $(20,21)$.

La experiencia de aprendizaje de los estudiantes de Enfermería en prácticas clínicas de salud mental puede ser positiva y educativa. El contar con espacio físico para actividades clínico-terapéuticas durante las prácticas clínicas puede influir en la percepción de los estudiantes respecto de las personas con enfermedad mental, con impacto positivo en el aprendizaje y en sus decisiones (22).

Asimismo, estudiantes después de terminar la práctica de enfermería en salud mental, han manifestado una mejoría en su confianza. Sin embargo, también han referido que requerían de mayor tiempo para practicar la comunicación terapéutica, administración de medicamentos y manejo de situaciones agudas. También señalaron que se sintieron menos seguros al evaluar: la medicación del cliente y su uso terapéutico, la educación al paciente y familia sobre la enfermedad psiquiátrica y la atención de acuerdo con los resultados previstos (23).

Respecto del rol de enfermero y docente clínico, manifiestan que se proporciona un 
aprendizaje efectivo, centrado en lo administrativo. Esta es una de las primeras instancias de aprendizaje en esta área, situación semejante a un estudio publicado en el Reino Unido que dirige la orientación hacia la primera experiencia de aprendizaje y que modela la adquisición de conocimiento al contexto administrativo (24).

Un aspecto importante de resaltar es el modelo conductista que se devela en los textos, contenido que igualmente se reproduce en otros estudios, como aprendizaje con orientación hacia el modelo biomédico (25). De esta forma se aprecian dificultades en la metodología y no se propicia al estudiante como protagonista de su propia formación y docente como ente facilitador.

Finalmente se observa un cambio desde el proceso de inicio a la finalización de sus prácticas, en lo relativo al miedo, nerviosismo o ansiedad, develándose ausencia de este al culminar las actividades de aprendizaje, situación similar a otro estudio $(9,8,26,27)$.

El proceso de aprendizaje en práctica clínica de salud mental y psiquiatría se considera esencial, productivo, importante, humano y necesario. La formación está permeada por el modelo conductista que permanece en el aprendizaje de los estudiantes, y la orientación biomédica, aun cuando la casa de estudio aboga por el constructivismo y el aprendizaje basado en problemas.

El ambiente de aprendizaje vivenciado por los estudiantes en el Servicio de Psiquiatría revela sensaciones como inquietud e inseguridad al enfrentarse a los pacientes, pero que con el transcurso de su permanencia en la práctica este sentimiento va desapareciendo.

Si bien el rol de enfermero y docente clínico proporciona un aprendizaje efectivo, su orientación se dirige netamente hacia el contexto administrativo.

Una de las actitudes que predomina en los estudiantes es estar a la defensiva por la estigmatización social que se revela frente a los pacientes, posteriormente la concepción so- bre ellos se modifica radicalmente, impregnándose en el estudiantado sentimientos de ayuda y comprensión de su situación de vida.

A través de esta investigación se espera instaurar situaciones de acercamiento de la teoría y práctica en los primeros años de la carrera de forma gradual en esta área, para limitar los temores que pueden presentar los estudiantes, con el propósito de optimar la adquisición de conocimientos en la práctica clínica que pueden estar restringidos por los sentimientos antes mencionados.

\section{REFERENCIAS}

1. Rodríguez J, Kohn R, Aguilar-Graxiola $\mathrm{S}$, eds. Epidemiología de los trastornos mentales en América Latina y el Caribe [Internet]. Washington, DC: Organización Panamericana de la Salud; 2009 Oct [citado 30 dic 2016]. Disponible en: http://apps.who.int/iris/bitstre am/10665/166275/1/9789275316320.pdf?ua $=1$

2. Kroning M. Strategies for Improving Nursing Students' Mental Health Clinical Rotation. J Christ Nurs. 2016 JulSep; 33(3): E31-7.

3. Vicente B, Rioseco P, Saldivia S, Kohn R, Torres S. Prevalencia de trastornos psiquiátricos en Latinoamérica: revisión crítica. Rev. Col. Psiquiatr. 2005; 34(4): 506.

4. Ministerio de Salud de Chile. Salud Mental. [Internet]. Santiago, Chile: Gobierno de Chile; 2015 Sep. [citado 7 ago 2015]. Disponible en: http://www.minsal.cl/salud-mental/

5. Happell B. Clinical experience in mental health nursing: determining satisfaction and the influential factors. Nurse Educ Today 2008; 28(7): 849-855.

6. Happell B. The importance of clinical experience for mental health nursing -part 2: relationships between undergraduate nursing students' attitudes, preparedness, 
and satisfaction. Int J Ment Health Nurs. 2008; 17(5): 333-40.

7. Vargas D, Oliveira MAF, Duarte FAB. Inclusión y prácticas del enfermero en los Centros de Atención Psicosocial para alcohol y drogas (CAPS AD) de la ciudad de Sao Paulo, Brasil. Rev Lat Am Enfermagem. 2011; 19(1): 115-122.

8. Henderson S, Happell B, Martin T. So what is so Good About Clinical Experience? A Mental Health Nursing Perspective. Nurse Educ Pract. 2007; 7(3): 164-172.

9. Rocha R, Cabrera E, González D, Martínez R, Pérez J, Saucedo R, et al. Factores de Estrés en Estudiantes Universitarios [Internet]. $11^{\circ}$ Congreso virtual de psiquiatría; febrero-marzo 2010. San Luis de Potosí, México: Psiquiatria.com; [citado 7 oct 2015]. Disponible en: http:// www.psiquiatria.com/bibliopsiquis/ assetstore/63/59/18/635918141 98252899557236892603040001977

10. Hernández A, Illesca M, Cabezas M. Opinión de estudiantes de la carrera de Enfermería Universidad Autónoma de Chile, Temuco, sobre las prácticas clínicas. Cienc enferm. 2013; XIX(1): 131-44.

11. Lima DWC, Silveira L, Vieira A, Cunha B, Almeida A, Guerreiro E. Referenciais teóricos que norteiam a prática de enfermagem em saúde mental. Esc Anna Nery. 2014; 18(2): 336-42.

12. Ayala Carabajo R. La Metodología Fenomenológico-hermenéutica de M. Van Manen en el Campo de la Investigación Educativa. Posibilidades y Primeras Experiencias. Revista de Investigación Educativa. 2008; 26(2): 409-30.

13. Taylor SJ, Bogdan R. Introducción a los Métodos Cualitativos de Investigación. La Búsqueda de Significados. Buenos Aires: Paidós; 1986. La observación participante. Preparación del trabajo de campo. p. 31-46.

14. Rodríguez G, Gil J, García E. Metodología de la Investigación Cualitativa. Málaga: Aljibe; 1996.378 p.
15. Dankhe OL. Investigación y comunicación. En: Fernández C, Dankhe G, eds. La comunicación humana: ciencia social. México, D.F: McGraw Hill; 1976. p. 384454.

16. Toro ID, Parra Ramírez RD. Método y Conocimiento. Metodología de la Investigación. Medellín: Fondo Editorial Universidad Eafit; 2006. 390 p.

17. Guba EG, Lincoln YS. Naturalistic Inquiry. London: Sage Publications; 1985. Designing a Naturalistic Inquiry. p 221250.

18. Colegio de Enfermeras de Chile. Código de Ética del Colegio de Enfermeras de Chile. [Internet]. Santiago, Chile: Colegio de Enfermeras de Chile; 2008 mayo [citado 7 ago 2015]. 19 p. Disponible en: http://enfermeriajw.com/wp-content/ uploads/2012/08/Coodigo-de-Etica-Colegio-de-Enfermeras-de-Chile.pdf

19. Asociación Médica Mundial (AMM). Declaración de Helsinki de la AMM Principios éticos para las investigaciones médicas en seres humanos [Internet]. 64a Asamblea General; 2013 oct. Fortaleza, Brasil: AMM; [citado 7 ago 2015]:8. Disponible en: http://www.wma.net/ es/30publications/10policies/b3/

20. Papastavrou E, Lambrinou E, Tsangari $\mathrm{H}$, Saarikoski M, Leino-Kilpi H. Student nurses experience of learning in the clinical environment. Nurse Educ Pract. 2010; 10(3): 176-182.

21. Grav S, Juul EML, Hellzén O. Undergraduate nursing student experiences of their mental health clinical placement. Nord J Nurs Res. 2010; 30(1): 4-8.

22. Patterson C, Moxham L, Brighton R, Taylor E, Sumskis S, Perlman D et al. Nursing students' reflections on the learning experience of a unique mental health clinical placement. Nurse Educ Today. 2016; 46: 94-98.

23. Fiedler RA, Breitenstein S, Delaney K. An assessment of students' confidence in performing psychiatric mental health 
nursing skills: the impact of the clinical practicum experience. J Am Psychiatr Nurses Assoc. 2012; 18(4): 244-50.

24. Andrew N. Clinical Imprinting: the impact of early clinical learning on career long professional development in nursing. Nurse Educ Pract 2013; 13(3): 161164.

25. Almeida AH, Soares CB. Educación en Salud: Análisis de la Enseñanza en la Graduación en Enfermería. Rev Lat Am Enfermagem 2011; 19(3): 1-8.
26. Beltrán RO, Scanlan JN, Hancock N, Luckett $\mathrm{T}$. The effect of first year mental health fieldwork on attitudes of occupational therapy students towards people with mental illness. Aust Occup Ther J. 2007; 54(1): 42-48.

27. Sharif F, Masoumi SA. Qualitative study of nursing student experiences of clinical practice. BMC Nurs [Internet]. 2005 [citado 7 ago 2015]; 4(6). Disponible en: http://bmcnurs.biomedcentral.com/articles/10.1186/1472-6955-4-6 